\title{
Clinical profiles and outcomes of pulmonary tuberculosis patients with delayed treatment at a tertiary hospital in South Korea
}

\author{
Sun-Hyung Kim ${ }^{1 \#}$, Jinsoo Min ${ }^{2 \#}$, Jun Yeun Cho ${ }^{1}$, Hyeran Kang ${ }^{1}$, Bumhee Yang ${ }^{1}$, Yoon Mi Shin ${ }^{1}$, \\ Kang Hyeon Choe ${ }^{1,3}, \mathrm{Ki}$ Man Lee $\mathrm{C}^{1,3}$ \\ ${ }^{1}$ Division of Pulmonary and Critical Care Medicine, Department of Internal Medicine, Chungbuk National University Hospital, Cheongju, Republic \\ of Korea; ${ }^{2}$ Division of Pulmonary and Critical Care Medicine, Department of Internal Medicine, Daejeon St. Mary's Hospital, College of Medicine, \\ The Catholic University of Korea, Seoul, Republic of Korea; ${ }^{3}$ Division of Pulmonary and Critical Care Medicine, Department of Internal Medicine, \\ College of Medicine, Chungbuk National University, Cheongju, Republic of Korea \\ Contributions: (I) Conception and design: J Min; (II) Administrative support: All authors; (III) Provision of study materials or patients: All authors; (IV) \\ Collection and assembly of data: SH Kim, J Min; (V) Data analysis and interpretation: SH Kim, J Min; (VI) Manuscript writing: All authors; (VII) \\ Final approval of manuscript: All authors. \\ \#These authors contributed equally to this work. \\ Correspondence to: Ki Man Lee, MD, PhD. Department of Internal Medicine, Chungbuk National University College of Medicine, 1 Chungdae-ro, \\ Seowon-gu, Cheongju, Chungbuk, 28644, Republic of Korea. Email: kimlee@chungbuk.ac.kr.
}

Background: Data on the clinical characteristics of delayed treatment initiation among pulmonary tuberculosis (TB) patients are lacking. Thus, this study aimed to identify the factors associated with delayed treatment in culture-confirmed pulmonary TB and to assess outcomes of delayed treatment.

Methods: We retrospectively evaluated 151 patients with culture-confirmed pulmonary TB between 2015 and 2017. Delayed and timely treatment was defined as initiation of anti-TB treatment after and before the identification of Mycobacterium tuberculosis complex isolate, respectively. Factors related to delayed treatment, such as comorbidities, clinical presentation, and patterns of initial healthcare use, were collected. We analyzed whether delayed treatment was associated with all-cause mortality using a multivariate binary logistic regression model adjusted for age, sex, cardiovascular disease, and malignancy.

Results: In total, 55 (36.4\%) patients had delayed treatment. The median length between the first medical visit and treatment initiation was 9 days. Compared with timely treatment, delayed treatment was associated with no initial visit to a non-pulmonary department [adjusted odds ratio (aOR) $=10.49,95 \%$ confidence interval (CI), 2.56-42.93] and absence of nucleic acid amplification test (aOR =7.54, 95\% CI, 2.75-20.67). After adjusting for age, sex, cardiovascular disease, and solid malignancies, delayed treatment was significantly associated with all-cause mortality ( $\mathrm{aOR}=3.79,95 \% \mathrm{CI}, 1.36-10.58)$. The most frequent possible cause of delayed treatment was the doctor's low suspicion of active TB disease.

Conclusions: Given that delayed treatment is associated with worse outcomes in South Korea, targeted interventions to increase awareness on TB in the healthcare community are necessary for additional mycobacterial tests and consults of suspicious patients to TB specialists.

Keywords: Mortality; diagnosis; healthcare; smear-negative; paucibacillary tuberculosis

Submitted Jul 31, 2020. Accepted for publication Dec 23, 2020.

doi: 10.21037/apm-20-1521

View this article at: http://dx.doi.org/10.21037/apm-20-1521 


\section{Introduction}

Tuberculosis (TB) is the leading cause of death from a single infectious agent worldwide, and its incidence rate remains high, with approximately 10.0 million incident cases recorded in 2018 (1). In addition to the treatment of existing cases, transmission should also be halted to end the global TB epidemic (2). In this context, early diagnosis and prompt initiation of treatment are among the most essential interventions to effectively prevent and control TB transmission. However, diagnosis and treatment delays are common worldwide, even in patients already found to have a positive sputum smear (3).

The cascade of care is a useful model for evaluating patient retention across sequential stages of care required to achieve a successful outcome (4). Several studies evaluating delays in the care cascade of TB showed that both patient and health system delays are influenced by various socioeconomic and clinical factors (5-7). For example, smear-negative pulmonary TB with atypical clinical presentations, especially among elderly patients, is often treated with fluoroquinolone, and its proper diagnosis and treatment is often delayed (8). Such delays in the diagnosis and treatment of TB might consequently impose a high risk of unfavorable outcome (9). Thus, the cause of delays should be addressed to improve TB control strategies.

Despite marked declines in the incidence of TB during the last several decades, South Korea continues to have the highest TB incidence among high-income countries. In addition, the incidence rate is rapidly increasing among those aged over 60 years as South Korea becomes an olderaged society, which imposes a huge obstacle on prompt TB diagnosis because of atypical clinical presentations of elderly patients with multiple comorbidities (10). Accordingly, several studies (11-13) have recently evaluated factors associated with delayed recognition and isolation of pulmonary TB among hospitalized patients in South Korea. However, data on the clinical characteristics of delayed treatment initiation among patients with pulmonary $\mathrm{TB}$ are still lacking. Thus, this study aimed to (I) describe each component of delays in the care cascade of TB among patients with culture-confirmed pulmonary TB, (II) identify factors associated with delayed treatment, and (III) assess the outcomes of delayed treatment.

We present the following article in accordance with the STROBE reporting checklist (available at http://dx.doi. org/10.21037/apm-20-1521).

\section{Methods}

\section{Study setting and subjects}

This retrospective study was conducted at Chungbuk National University Hospital, which is a university-affiliated tertiary hospital that participates in the public-private mix (PPM) TB control project. We evaluated patients with culture-conformed pulmonary TB between November 2015 and March 2017. Of the 366 patients initially identified, 215 patients with only extrapulmonary involvement $(n=90)$ and those with negative or unavailable acid-fast bacilli (AFB) culture test results $(n=125)$ were excluded. Finally, 151 patients were included in the analysis. They were divided into timely and delayed treatment groups according to if anti-TB treatment was initiated before or after the identification of Mycobacterium tuberculosis complex isolate from the AFB culture test, respectively. All TB patients underwent a 6-month treatment regimen as recommended by the National Tuberculosis Program. Anti-TB drugs were self-administered with the support of the PPM nurses. The study was approved by the Institutional Review Board of Chungbuk National University Hospital, South Korea (IRB No. 2020-02-022) and was conducted in accordance with the amended Declaration of Helsinki (as revised in 2013). The need for informed consent was waived because no patients were at risk.

\section{Data collection}

Data on demographics, comorbidities, and clinical presentation were retrospectively collected from the medical records. The results of the initial microbiological tests were also obtained. Radiographic and computed tomography (CT) findings were classified as active lesions suggestive of TB, active lesions suggesting disease conditions other than $\mathrm{TB}$, and inactive lesions. Data regarding patterns of initial healthcare use that might be associated with delayed antiTB treatment, such as initial route of hospital visit and types of initial microbiological and radiological tests, were also collected. Treatment outcomes were classified as cure, treatment completion, treatment failure, loss to follow-up, and death following the World Health Organization (WHO) recommendations.

\section{Definition of delays of TB care cascade}

To describe the days elapsed from symptom onset to the 


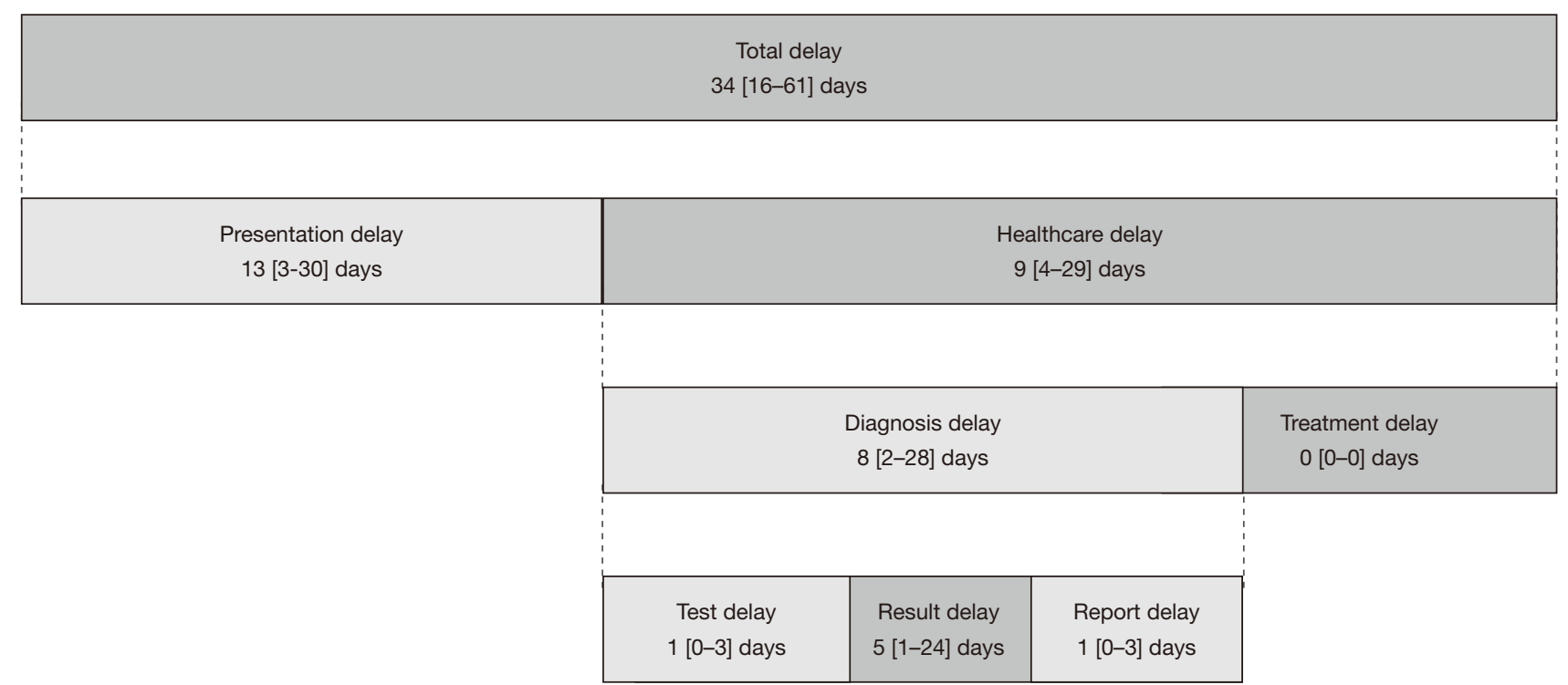

Figure 1 Components of delays in diagnosis and treatment among culture-confirmed pulmonary tuberculosis patients and their median length with interquartile range.

first medical visit, diagnosis, and treatment, the delays were defined and categorized based on a previous study (6). Presentation delay was defined as the interval between onset of symptoms suggestive of pulmonary TB and the patient's first visit to clinic (Figure 1). Diagnosis delay was defined as the interval between the first medical visit and TB diagnosis. Treatment delay was defined as the interval between TB diagnosis and initiation of anti-TB treatment. Healthcare delay was defined as the sum of diagnosis and treatment delay, whereas total delay was defined as the sum of presentation delay and healthcare delay. Diagnosis delay was further divided into 3 subcategories as test, result, and report delays. Test delay was defined as the interval between the first medical visit and microbiological testing. Result delay was defined as the interval between microbiological testing and the first report of a test result suggestive of TB. Report delay was defined as the interval between the first report of a test result and the doctor's first recognition of a test result that led to the diagnosis of TB.

\section{Statistical analysis}

Discrete variables are presented as frequency or percentage. The distribution of delays from symptom onset to treatment initiation was expressed as median and interquartile range (IQR). Between-group comparisons were conducted using univariate analysis via the chi-square test and binary logistic regression. We hypothesized that delayed treatment would be associated with comorbidities, clinical presentation, and patterns of initial healthcare use. Accordingly, clinical variables with a $\mathrm{P}$ value of $<0.20$ on univariate analysis were selected in addition to age and sex and included in the multivariate binary logistic regression to evaluate the possible association between variables and delayed treatment. Multivariate binary logistic regression analysis adjusted for cardiovascular disease and malignancy as leading causes of death in Korea (37.2\%) in 2018 (14) was also conducted to evaluate the association between delayed treatment and treatment outcome. Statistical analyses were performed using SPSS version 21.0 (IBM Corp., Armonk, NY, USA), and $\mathrm{P}<0.05$ was considered statistically significant.

\section{Results}

\section{Patient characteristics}

There were 85 (56.3\%) patients aged $\geq 65$ years, and 92 (60.9\%) male patients. The most common symptoms were cough $(50.3 \%)$ and sputum production (45.7\%). In total, 96 $(63.6 \%)$ and $55(36.4 \%)$ patients received timely and delayed treatment, respectively. Their baseline characteristics are shown in Table 1. The delayed treatment group had 
Table 1 Clinicodemographic patient characteristics

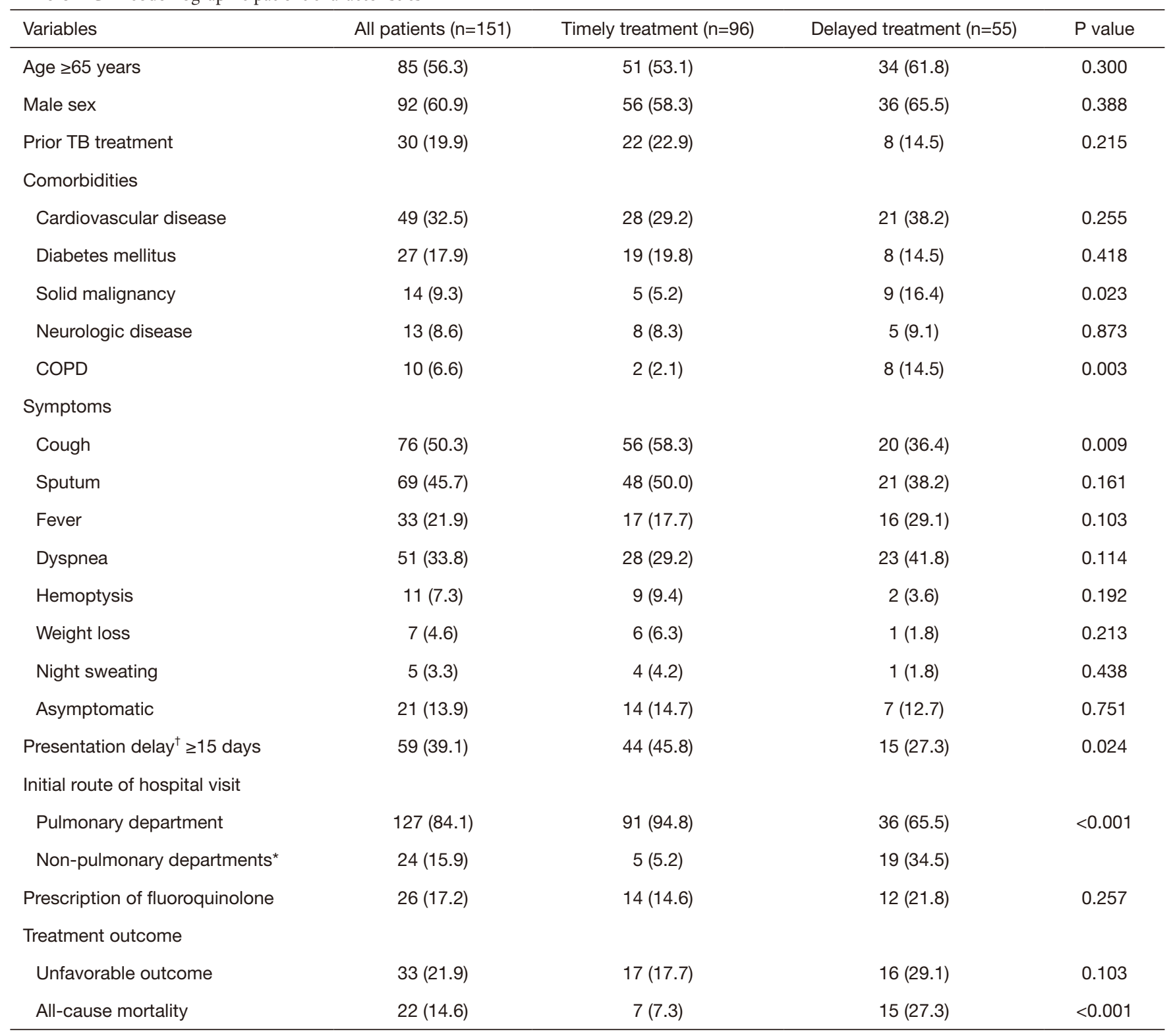

Data are presented as number (\%). *, non-pulmonary departments include neurology, emergency department, general surgery, nephrology, and cardiology. ${ }^{\dagger}$, presentation delay was defined as the interval between onset of symptoms suggestive of pulmonary TB and the patient's first visit to the clinic. TB, tuberculosis; COPD, chronic obstructive pulmonary disease; HIV, human immunodeficiency virus; ICU, intensive care unit.

a significantly higher rate of solid malignancy $(16.4 \% \mathrm{vs}$. $5.2 \%, \mathrm{P}=0.023)$ and chronic obstructive pulmonary disease ( $14.5 \%$ vs. $2.1 \%, \mathrm{P}=0.003)$ than the timely treatment group. The proportion of patients with presentation delay $\geq 15$ days was significantly lower in the delayed treatment group compared to the timely treatment group $(27.3 \% v s$. $45.8 \%, \mathrm{P}=0.024)$. The rate of initial visit to the pulmonary department was significantly lower in the delayed treatment group than that in the timely treatment group $(65.5 \% \mathrm{vs}$. $94.8 \%, \mathrm{P}<0.001)$. Meanwhile, the proportion of patients with prior use of fluoroquinolone before $\mathrm{TB}$ diagnosis was similar between the two groups $(21.8 \%$ vs. $14.6 \%, \mathrm{P}=0.257)$. Mortality was significantly higher in the delayed treatment group $(27.3 \%$ vs. $7.3 \%, \mathrm{P}<0.001)$. 
Table 2 Laboratory findings

\begin{tabular}{|c|c|c|c|c|}
\hline Variables & All patients $(n=151)$ & Timely treatment $(n=96)$ & Delayed treatment $(n=55)$ & $P$ value \\
\hline Positive & $34(22.5)$ & $34(35.4)$ & $0(0)$ & $<0.001$ \\
\hline Negative & $117(77.5)$ & $62(64.6)$ & $55(100.0)$ & \\
\hline \multicolumn{5}{|c|}{ Nucleic acid amplification test } \\
\hline Negative & $46(30.5)$ & $25(26.0)$ & $21(38.2)$ & \\
\hline Not performed & $14(29.1)$ & $14(14.6)$ & $30(54.5)$ & \\
\hline \multicolumn{5}{|c|}{ Number of AFB tests performed } \\
\hline Once & $42(27.8)$ & $21(21.9)$ & $21(38.2)$ & 0.031 \\
\hline \multicolumn{5}{|l|}{ Initial radiographic findings } \\
\hline No active lung lesions & $9(6.0)$ & $4(4.2)$ & $5(9.1)$ & 0.219 \\
\hline Active TB disease & $79(52.3)$ & $62(64.6)$ & $17(30.9)$ & $<0.001$ \\
\hline Pneumonia & $42(27.8)$ & $18(18.8)$ & $24(43.6)$ & 0.001 \\
\hline Lung cancer & $3(2.0)$ & $1(1.0)$ & $2(3.6)$ & 0.272 \\
\hline Pulmonary nodule & $7(4.6)$ & $3(3.1)$ & $4(7.3)$ & 0.243 \\
\hline Pleural effusion & $25(16.6)$ & $14(14.6)$ & $11(20.0)$ & 0.389 \\
\hline
\end{tabular}

AFB, acid-fast bacilli; TB, tuberculosis; CT, computerized tomography.

The laboratory findings are shown in Table 2. In total, $22.5 \%(34 / 151)$ and $57.0 \%(61 / 107)$ of the patients had positive results on the AFB smear and nucleic acid amplification test (NAAT). The proportion of patients who underwent the AFB culture test more than twice was significantly higher in the timely treatment group than in the delayed treatment group $(78.1 \%$ vs. $61.8 \%, \mathrm{P}=0.031)$. The proportion of patients who underwent NAAT was significantly higher in the timely treatment group than that in the delayed treatment group $(85.4 \%$ vs. $45.5 \%, \mathrm{P}<0.001)$. A review of chest imaging findings showed that the most common finding was active TB $(52.3 \%)$, followed by pneumonia (27.8\%) and pleural effusion (16.6\%).

\section{Characteristics and influencing factors of delay}

Each component of delay in the care cascade of TB is shown in Figures 1,2. The median length from symptom onset to initiation of anti-TB treatment was 34 days, with an average of 41.2 days. The median length of presentation delay was 13 days, with average of 21.5 days. The median length of healthcare delay was 9 days, with an average of 19.7 days. Test delay ranged from 0 to 141 days, with a median of 1 day (IQR: $0-3$ days). Result delay ranged from 0 to 78 days, with a median of 5 days (IQR: $1-1$ days). Report delay ranged from 0 to 18 days, with a median of 1 day (IQR: $0-3$ days). Treatment delay ranged from 0 to 64 days, with a median of 0 day (IQR: $0-0$ day).

Delayed treatment was associated with no initial visit to a non-pulmonary department [adjusted odds ratio $(\mathrm{aOR})=10.49,95 \%$ confidence interval $(\mathrm{CI}), 2.56-42.93]$ and NAAT ( $\mathrm{aOR}=7.54,95 \% \mathrm{CI}, 2.75-20.67$ ) (Table 3). Although the aORs of chronic obstructive pulmonary disease and no chest CT were 6.55 (95\% CI, 0.96-44.70) and 11.25 (0.68-189.98), respectively, they were not significant.

With respect to possible causes of delayed treatment, the most frequent was the doctor's low suspicion of active TB disease $(58.2 \%, 32 / 55)$ and an initial diagnosis other than $\mathrm{TB}$, such as bacterial pneumonia, upper respiratory 


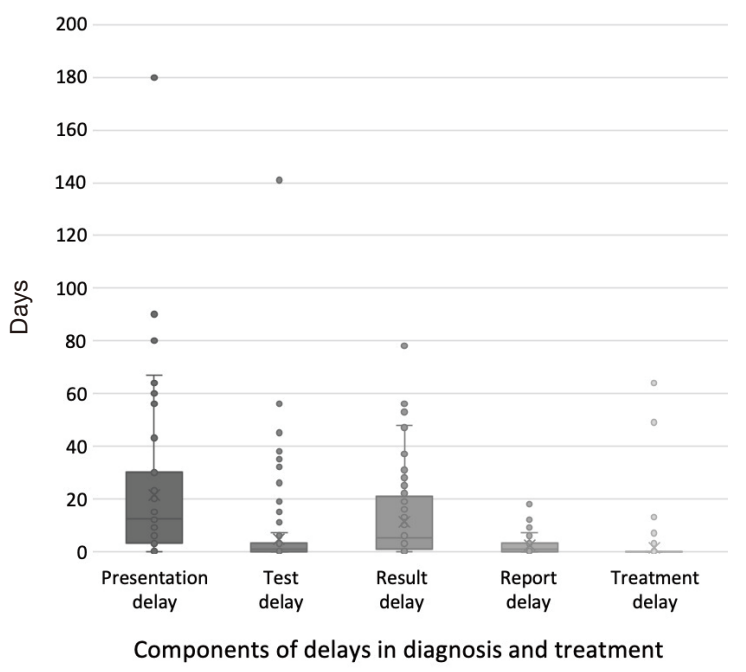

Figure 2 Box plots of delays in diagnosis and treatment among culture-confirmed pulmonary tuberculosis patients.

infection, or advanced lung cancer. There were 12 patients (21.8\%) diagnosed with inactive TB diseases based on negative results of the AFB smear test and NAAT and initial radiographic findings. Eight patients (14.5\%) did not undergo additional tests to confirm the diagnosis of TB. Initial diagnosis was missed in $3(5.5 \%)$ patients as they did not revisit the clinic to check positive results of microbiological tests.

Multivariate analysis of the factors associated with all-cause mortality showed that delayed treatment was significantly associated with all-cause mortality (aOR $=4.30,95 \%$ CI, 1.59-11.61) after adjusting for age and sex (Table 4). After further adjusting for cardiovascular disease and solid malignancy, the odds ratio of all-cause mortality was 3.79 (95\% CI, 1.36-10.58). In total, 8 of the 15 mortality cases in the delayed treatment group were postmortem cases (Table 5).

\section{Discussion}

Data on the clinical characteristics of delayed treatment initiation among pulmonary TB patients in Korea are still lacking. Delayed TB treatment in the Korean population is frequently caused by a lack of clinical consult and no NAAT and is associated with worse outcomes, including higher all-cause mortality. Early diagnosis and prompt initiation of treatment are among the most essential interventions for an effective TB control program. In 2011, South Korea
Table 3 Multivariable logistic regression analyses of factors associated with delayed treatment

\begin{tabular}{lcc}
\hline Variables & aOR $(95 \% \mathrm{Cl})$ & $\mathrm{P}$ value \\
\hline Age $\geq 65$ years & $1.02(0.38-2.73)$ & 0.969 \\
Male sex & $1.15(0.41-3.17)$ & 0.793 \\
COPD & $6.55(0.96-44.70)$ & 0.055 \\
Solid malignancy & $2.44(0.46-13.03)$ & 0.296 \\
Cough & $0.35(0.08-1.54)$ & 0.164 \\
Sputum & $1.44(0.31-6.70)$ & 0.638 \\
Fever & $1.16(0.39-3.47)$ & 0.792 \\
Dyspnea & $2.52(0.85-7.42)$ & 0.094 \\
Hemoptysis & $0.84(0.11-6.44)$ & 0.866 \\
Presentation delay $\geq 15$ days & $0.41(0.15-1.11)$ & 0.080 \\
Initial visit to non-pulmonary & $10.49(2.56-42.93)$ & 0.001 \\
department & & \\
AFB testing performed only & $1.93(0.73-5.07)$ & 0.186 \\
once & & \\
NAAT not performed & $7.54(2.75-20.67)$ & $<0.001$ \\
Chest CT not performed & $11.25(0.68-189.98)$ & 0.091 \\
\hline
\end{tabular}

aOR, adjusted odds ratio; $\mathrm{Cl}$, confidence interval; COPD, chronic obstructive pulmonary disease; AFB, acid-fast bacilli; NAAT, nucleic acid amplification test; CT, computerized tomography.

implemented the national PPM TB control project that provides comprehensive patient management, helping increase the treatment success rate and decreasing the incidence of TB (15). However, there are limited studies evaluating the accuracy and timing of diagnosis before initiation of treatment in the PPM era. A previous Korean study (16) that evaluated 107 pulmonary TB patients who visited a tertiary university hospital between 2011 and 2012 reported an average of 16.7 and 22.2 days for patient and doctor delays, respectively. From a public health perspective, it is important to periodically assess any delays in diagnosis and treatment as this will increase awareness of the problems in TB management. For example, improving access to care and ensuring early diagnosis are key areas for action as defined in the national TB strategy in the United Kingdom. Further, the proportion of pulmonary TB patients starting treatment within 2 to 4 months of symptom onset are important monitoring indicators (17).

We hypothesized that treatment delay was associated with diagnostic modalities and methods and retrospectively 
Table 4 Multivariate logistic regression analysis of factors associated with all-cause mortality

\begin{tabular}{|c|c|c|c|c|}
\hline Variables & aOR $(95 \% \mathrm{Cl})$ & $P$ value & aOR (95\% Cl) & $P$ value \\
\hline Age $\geq 65$ years & $2.87(0.96-8.56)$ & 0.058 & $1.88(0.56-6.28)$ & 0.588 \\
\hline Male sex & $2.32(0.77-6.97)$ & 0.134 & $1.65(0.51-5.36)$ & 0.409 \\
\hline Cardiovascular disease & & & $1.95(0.67-5.71)$ & 0.224 \\
\hline
\end{tabular}

$\mathrm{Cl}$, confidence interval; aOR, adjusted odds ratio.

Table 5 Characteristics of patients diagnosed with pulmonary tuberculosis after death

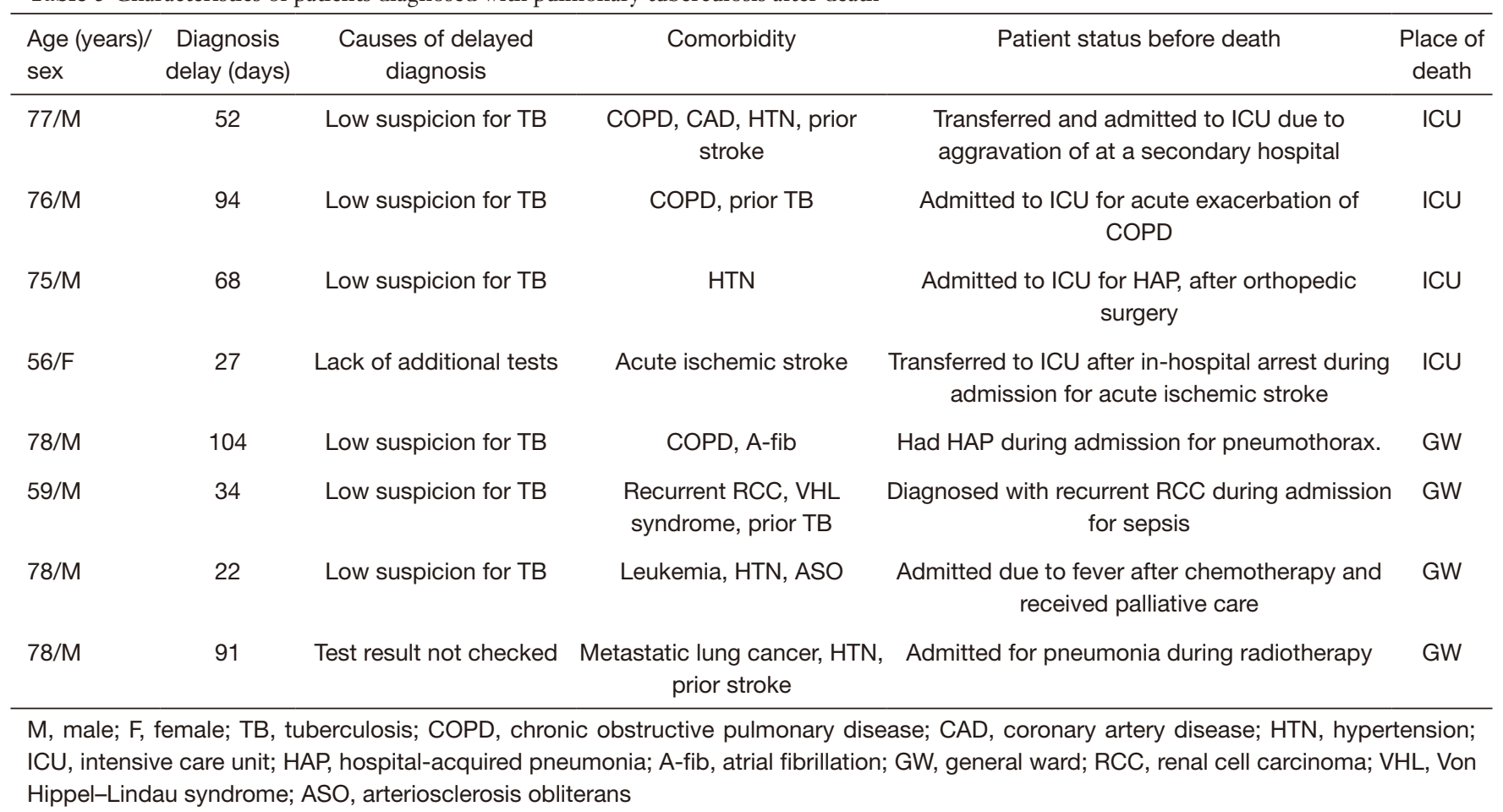

collected related data. The proportion of patients with an initial hospital visit to a non-pulmonary department was significantly higher in the delayed treatment group. Clinicians in departments other than pulmonology and infectious diseases sometimes miss or ignore a positive report of TB tests, resulting in diagnosis and treatment delays (18). A low suspicion of TB might hinder the performance of additional microbiological tests or consulting a pulmonologist despite chest imaging findings of active TB disease. A multifaceted approach to increase clinician awareness of the clinical presentations of $\mathrm{TB}$ is necessary to actively identify TB.
In the current study, not undergoing NAAT was significantly associated with delayed treatment. This finding is reasonable given that NAAT is an important diagnostic modality for TB, with better accuracy and faster turnaround time than the AFB smear test. A recent study suggested that the Xpert MTB/RIF assay (Cepheid, Sunnyvale, CA, USA) might replace smear microscopy as the first-line diagnostic test for pulmonary $\mathrm{TB}$ in routine clinical practice in an intermediateburden setting (19). The number of NAATs markedly increased between 2007 and 2014 in South Korea (20). The updated Korean TB guidelines in 2017 removed the 
limit of NAAT and broadened its use for suspected TB cases (21). About one-third of the enrolled patients in the current study did not undergo NAAT or underwent AFB testing only once. All patients in the delayed treatment group were smear-negative, implying paucibacillary disease. Importantly, a negative sputum smear is known to be associated with a longer healthcare delay (22). In addition to increasing clinician awareness, performing prompt and appropriate diagnostic tests is essential to avoid a missed TB diagnosis.

Nearly all the enrolled patients had chest CT performed during the initial hospital visit, and additional usage of chest CT scans was not different between the timely and delayed treatment groups. Forty-two patients had initial chest imaging findings suggestive of bacterial pneumonia, and they were accordingly initially treated with antibiotics. Among them, 61.9\% (26/42) were prescribed with fluoroquinolone before TB diagnosis. Although chest CT and the prescription of fluoroquinolone were not significant factors, a previous study showed that anti-TB treatment may be delayed in patients administered fluoroquinolone and initially misdiagnosed with bacterial pneumonia (23). When prescribing fluoroquinolone for bacterial pneumonia in high- or intermediate-burden settings, it is important to assess the resolution of pneumonia after antimicrobial therapy and identify results of mycobacterial tests.

Our study showed that delayed treatment was significantly associated with all-cause mortality after adjusting for sex, age, cardiovascular disease, and solid malignancy. This finding might be attributed to the postmortem TB cases. Most of them were old-aged patients with multiple morbidities, and delayed diagnosis ranged from 22 to 104 days. Because these patients initially visited the hospital because of other acute illnesses, our analysis of the post-mortem patients showed a low suspicion of TB disease and smear negativity, similar to the findings of a previous study (24).

This study has some limitations. First, we could not evaluate the factors associated with presentation delay, which is an important contributing factor to $\mathrm{TB}$ transmission. Second, presentation delays were determined retrospectively, and they could have been limited by recall bias. Third, because of the retrospective study design, we could not collect data on visits to other healthcare facilities between symptom onset and the patient's visit to the study hospital. Fourth, the study included only a small number of patients from a single tertiary hospital, thus limiting the generalizability of our findings. Because we used low events- per-variable ratios, a multivariable model describing factors associated with delayed treatment might become unstable. Further larger studies are necessary to confirm our result. Additional studies evaluating presentation and healthcare delays among patients with culture-negative and clinically diagnosed TB are necessary. In addition, the diagnostic pathway for TB patients needs to be evaluated to further understand the barriers to diagnosis and treatment. For example, socioeconomic status is an important determinant of healthcare-seeking behavior, which might influence our results. Further in-depth qualitative studies evaluating physicians' attitude and knowledge could identify causes of healthcare delay.

In conclusion, treatment delays for TB in South Korea are primarily due to the physician's low suspicion of active TB disease and an initial diagnosis other than TB. The absence of NAAT also contributed to a delay in treatment. Importantly, treatment delay is associated with all-cause mortality. These findings support the need for targeted interventions to increase awareness on TB and improve treatment delays.

\section{Acknowledgments}

Funding: This work was supported by the research grant of the Chungbuk National University Hospital in 2020.

\section{Footnote}

Reporting Checklist: The authors have completed the STROBE reporting checklist. Available at http://dx.doi. org/10.21037/apm-20-1521

Data Sharing Statement: Available at http://dx.doi. org/10.21037/apm-20-1521

Conflicts of Interest: All authors have completed the ICMJE uniform disclosure form (available at http://dx.doi. org/10.21037/apm-20-1521). Dr. SHK reports grants from the research grant of the Chungbuk National University Hospital, during the conduct of the study. The other authors have no conflicts of interest to declare.

Ethical Statement: The authors are accountable for all aspects of the work in ensuring that questions related to the accuracy or integrity of any part of the work are appropriately investigated and resolved. The study was approved by the Institutional Review Board of Chungbuk 
National University Hospital, South Korea (IRB No. 202002-022) and was conducted in accordance with the amended Declaration of Helsinki (as revised in 2013). The need for informed consent was waived because no patients were at risk.

Open Access Statement: This is an Open Access article distributed in accordance with the Creative Commons Attribution-NonCommercial-NoDerivs 4.0 International License (CC BY-NC-ND 4.0), which permits the noncommercial replication and distribution of the article with the strict proviso that no changes or edits are made and the original work is properly cited (including links to both the formal publication through the relevant DOI and the license). See: https://creativecommons.org/licenses/by-nc-nd/4.0/.

\section{References}

1. World Health Organization. Global Tuberculosis Report 2019 Geneva: World Health Organization; 2019.

2. Yuen CM, Amanullah F, Dharmadhikari A, et al. Turning off the tap: stopping tuberculosis transmission through active case-finding and prompt effective treatment. Lancet 2015;386:2334-43.

3. Davis J, Katamba A, Vasquez J, et al. Evaluating tuberculosis case detection via real-time monitoring of tuberculosis diagnostic services. Am J Respir Crit Care Med 2011;184:362-7.

4. Subbaraman R, Nathavitharana RR, Mayer KH, et al. Constructing care cascades for active tuberculosis: A strategy for program monitoring and identifying gaps in quality of care. PLoS Med 2019;16:e1002754.

5. Finnie RK, Khoza LB, van den Borne B, et al. Factors associated with patient and health care system delay in diagnosis and treatment for TB in sub-Saharan African countries with high burdens of TB and HIV. Trop Med Int Health 2011;16:394-411.

6. Sreeramareddy CT, Qin ZZ, Satyanarayana S, et al. Delays in diagnosis and treatment of pulmonary tuberculosis in India: a systematic review. Int J Tuberc Lung Dis 2014;18:255-66.

7. Cai J, Wang X, Ma A, et al. Factors associated with patient and provider delays for tuberculosis diagnosis and treatment in Asia: a systematic review and meta-analysis. PLoS One 2015;10:e0120088.

8. Zhang ZX, Sng LH, Yong Y, et al. Delays in diagnosis and treatment of pulmonary tuberculosis in AFB smear- negative patients with pneumonia. Int J Tuberc Lung Dis 2017;21:544-9.

9. Asres A, Jerene D, Deressa W. Delays to treatment initiation is associated with tuberculosis treatment outcomes among patients on directly observed treatment short course in Southwest Ethiopia: a follow-up study. BMC Pulm Med 2018;18:64.

10. Min J, Mi Shin Y, Lee WJ, et al. Clinical features of octogenarian patients with tuberculosis at a tertiary hospital in South Korea. J Int Med Res 2019;47:271-80.

11. Han J, Nam BD, Park SY, et al. Risk Factors for Delayed Isolation of Patients with Active Pulmonary Tuberculosis in an Acute-care Hospital. Sci Rep 2019;9:4849.

12. Heo DH, Seo JW, Kim JH, et al. Delays in Isolating Patients Admitted to Hospital with Pulmonary Tuberculosis in Korea. J Korean Med Sci 2019;34:e270.

13. Kim CJ, Kim Y, Bae JY, et al. Risk factors of delayed isolation of patients with pulmonary tuberculosis. Clin Microbiol Infect 2020;26:1058-62.

14. Statistics Korea. Causes of death statistics in 2018. Available online: https://www.kostat.go.kr/portal/korea/ kor_nw/1/1/index.board?bmode=read\&aSeq=377606

15. Cho KS. Tuberculosis control in the Republic of Korea. Epidemiol Health 2018;40:e2018036.

16. Park JS. Symptoms and Factors Associated with Delayed Diagnosis of Pulmonary Tuberculosis. Korean J Med 2013;84:221-8.

17. Lipman M, White J. Collaborative tuberculosis strategy for England. BMJ 2015;350:h810.

18. Chen TC, Lu PL, Lin WR, et al. Diagnosis and treatment of pulmonary tuberculosis in hospitalized patients are affected by physician specialty and experience. Am J Med Sci 2010;340:367-72.

19. Lee HS, Kee SJ, Shin JH, et al. Xpert MTB/RIF Assay as a Substitute for Smear Microscopy in an Intermediate-Burden Setting. Am J Respir Crit Care Med 2019;199:784-94.

20. Kim CK, Choi SW, Park MS. Utilization of laboratory tests for tuberculosis and mycobacterial disease in Korea. Osong Public Health Res Perspect 2014;5:S24-9.

21. Joint Committee for the Revision of Korean Guidelines for Tuberculosis, Korea Centers for Disease Control and Prevention. Korean Guidelines for Tuberculosis. 4th ed. Seoul and Cheongwon: Joint Committee for the Revision of Korean Guidelines for Tuberculosis, Korea Centers for Disease Control and Prevention; 2020.

22. Roberts DJ, Mannes T, Verlander NQ, et al. Factors 
associated with delay in treatment initiation for pulmonary tuberculosis. ERJ Open Res 2020;6:00161-2019.

23. Yoon YS, Lee HJ, Yoon HI, et al. Impact of fluoroquinolones on the diagnosis of pulmonary tuberculosis initially treated as bacterial pneumonia. Int J

Cite this article as: Kim SH, Min J, Cho JY, Kang H, Yang B, Shin YM, Choe KH, Lee KM. Clinical profiles and outcomes of pulmonary tuberculosis patients with delayed treatment at a tertiary hospital in South Korea. Ann Palliat Med 2021;10(3):29482957. doi: 10.21037/apm-20-1521
Tuberc Lung Dis 2005;9:1215-9.

24. Leung EC, Leung CC, Chang KC, et al. Delayed diagnosis of tuberculosis: risk factors and effect on mortality among older adults in Hong Kong. Hong Kong Med J 2018;24:361-8. 